\title{
Projeto de um Reator de Amônia Usando o Whale Optimization Algorithm
}

\section{Design of an Ammonia Reactor using the Whale Optimization Algorithm}

Ozanan Garcia Mundim Filho', Fran Sérgio Lobato², Davi Leonardo de Souza ${ }^{3}$

\section{RESUMO}

O presente estudo tem por objetivo a determinação de condições de operação (o comprimento do reator, o número de moles de nitrogênio, a temperatura do gás de reação e a temperatura do gás de alimentação) de um reator de amônia através da maximização do lucro. Para essa finalidade é empregado uma nova estratégia bioinspirada na natureza, a saber, o Whale Optimization Algorithm, que é inspirado no comportamento social de baleias jubarte. Além disso, considera-se uma nova estratégia para a atualização dinâmica de parâmetros deste algoritmo através do conceito de taxa de convergência. Os resultados obtidos demonstraram a habilidade da metodologia auto-adaptativa proposta para o projeto do reator de amônia.

Palavras-chave: Reator de amônia, otimização, Taxa de convergência, Whale Optimization Algorithm.

\section{ABSTRACT}

The aim of this study was to determine the operating conditions (reactor length, number of nitrogen moles, reaction gas temperature and feed gas temperature) of an ammonia reactor through the maximization of profit. To solve this problem, a new bioinspired strategy is considered, the Whale Optimization Algorithm, which mimics the social behavior of humpback whales. In addition, a new strategy to update the parameters in this algorithm considering the concept of convergence rate is proposed. The results obtained demonstrated the ability of the proposed self-adaptive methodology to design of an ammonia reactor.

Keywords: Ammonia reactor, Optimization, Convergence Rate, Whale Optimization Algorithm.

1 Programa de Pós-Graduação em Modelagem e Otimização,

Universidade Federal de Goiás, Unidade Catalão, GO, Brasil.

E-mail: ozanan12@hotmail.com

${ }^{2}$ Faculdade de Engenharia

Química, Universidade Federal de Uberlândia, Uberlândia, MG, Brasil.

${ }^{3}$ Departamento de Engenharia Química, Universidade Federal do Triângulo Mineiro, Uberaba, MG, Brasil. 


\section{INTRODUÇĀO}

Em engenharia química, o projeto de sistemas caracteriza-se como uma área de pesquisa com grande aplicabilidade no meio acadêmico e na indústria. Em linhas gerais, pode-se dizer que estes problemas apresentam grande complexidade devido aos modelos diferenciais e algébricos não lineares que são empregados para a representação dos fenômenos envolvidos em um determinado processo. Neste cenário, o projeto relacionado a estes modelos complexos representa um grande desafio para os projetistas.

Dentre as aplicações em engenharia química que podem ser encontradas na literatura especializada, a síntese de amônia caracteriza-se como um processo extremamente importante visto a sua aplicabilidade nas indústrias de fertilizantes, produtos químicos, explosivos, fibras, plásticos, refrigeração, produtos farmacêuticos, celulose, papel, mineração, metalurgia e limpeza.

Amônia é produzida a partir da reação exotérmica reversível de hidrogênio e nitrogênio $\left(\mathrm{N}_{2}+3 \mathrm{H}_{2} \leftrightarrow \mathrm{NH}_{3}-92 \mathrm{KJ} / \mathrm{mol}\right)$ a temperatura e pressão elevadas e na presença de um catalisador (MURASE et al., 1970; UPRETI e DEB, 1997; EDGAR e HIMMELBLAU, 1989). Devido à alta pressão requerida, o consumo energético associado a este processo é elevado, o que torna mais rigorosas as exigências de redução de custos e de aumento de rendimento. Além disso, para aumentar a resistência do reator a altas pressões, a sua parede tem configuração especial (em sua fabricação, de ligas de certos materiais) (EDGAR e HIMMELBLAU, 1989).

$\mathrm{Na}$ literatura, inúmeros trabalhos abordando a modelagem, a simulação e a otimização mono-objetivo do reator de síntese auto-térmica de amônia podem ser encontrados. Annable (1952) propôs uma estratégia para o controle de temperatura no reator de síntese auto-térmica de amônia. Dyson (1965) maximizou o rendimento de um reator auto térmico utilizado para a síntese de amônia através da determinação do coeficiente de transferência de calor em função do comprimento. Murase et al. (1970) determinou para o mesmo sistema o coeficiente global de transferência de calor. Edgar e Himmelblau (1989), utilizando o Método do Gradiente Generalizado de Lasdon, determinaram o lucro máximo através da otimização do comprimento de um reator de síntese de amônia. Entretanto, estes autores utilizaram expressões incorretas para as pressões parciais de nitrogênio, hidrogênio e amônia, necessárias para a simulação do perfil de temperatura e dos fluxos ao longo do comprimento do reator. Upreti e Deb (1997) apresentaram um procedimento para a maximização do lucro em um reator de síntese de 
amônia usando algoritmos genéticos associado a subrotina NAG (Numerical Algorithm Group) para a simulação do modelo matemático. No entanto, estes autores, diferentemente de Edgar e Himmelblau (1989), utilizaram as expressões corretas para as pressões parciais de $\mathrm{N}_{2}, \mathrm{H}_{2}, \mathrm{NH}_{3}$. Como principais resultados pode-se destacar a obtenção dos perfis de fluxo de massa de hidrogênio, da temperatura do gás de alimentação, e da temperatura do gás de reação em função do comprimento do reator. Entretanto, observa-se uma contradição nos perfis das temperaturas e do fluxo de massa obtidos e uma condição de reação inversa sob temperaturas superiores a $664 \mathrm{~K}$, o que não foi encontrado em nenhum outro trabalho da literatura. Babu et al. (2004) e Babu e Angira (2005) resolveram o problema estudado por Upreti e Deb (1997). Todavia, estes autores conseguiram demostrar que a condição de reação inversa citada não existia, sendo que esta incoerência estava relacionada com o tipo de solver numérico utilizado por Upreti e Deb (1997) para a simulação do modelo matemático. Yusup et al. (2006) e Ksasy et al. (2006), de posse das correções apresentadas no modelo e no algoritmo de simulação, demonstraram que o resultado obtido por Upreti e Deb (1997) não representa uma condição ótima de operação. Borges et al. (2012) propuseram a otimização de um reator auto-térmico para a síntese de amônia usando o Algoritmo de Busca Gravitacional considerando os aspectos acima mencionados nas etapas de modelagem e simulação.

Como observado, inúmeras abordagens (clássicas ou heurísticas) já foram propostas para resolver este estudo de caso. O presente trabalho tem por objetivo determinar o comprimento do reator, o fluxo molar de nitrogênio, a temperatura do gás de reação, e a temperatura do gás de alimentação, para uma dada temperatura de referência de modo a maximizar o lucro com o processo. Para essa finalidade será considerado uma nova estratégia de otimização bio-inspirada, a saber, o Whale Optimization Algorithm WOA (MIRJALILI e LEWIS, 2016). Em linhas gerais, este novo algoritmo de otimização é inspirado no comportamento social de baleias jubarte para a geração de candidatos a solução do problema de otimização. Cabe ressaltar que uma nova estratégia para a atualização dinâmica de alguns parâmetros deste algoritmo, através do conceito de taxa de convergência, é proposta. Este trabalho está estruturado como segue: a seção 2 apresenta a modelagem matemática para fins do projeto de um reator de síntese autotérmica de amônia; já na seção 3 é apresentada a descrição do WOA; nas seções 4 e 5 são apresentados a metodologia proposta e os resultados, respectivamente. Finalmente, as conclusões são mostradas na última seção. 


\section{MODELAGEM MATEMATICA DO PROCESSO}

A Figura 1 apresenta o diagrama esquemático do reator auto-térmico de amônia considerado nesta contribuição. O gás de alimentação com nitrogênio $(21,75 \%$ em moles), hidrogênio ( $62,25 \%$ em moles), amônio ( $5 \%$ em moles), metano ( $4 \%$ em moles) e argônio ( $4 \%$ em moles) entra pelo fundo do reator e é pré-aquecido pelo gás que entra em contra-corrente para fins da promoção da reação exotérmica reversível no catalisador, onde a amônia é produzida. O gás reagido, que consiste no nitrogênio e hidrogênio não convertidos, além de amônia e inertes, irá reagir com o gás de alimentação antes de deixar o reator.

Figura 1. Diagrama esquemático de um reator de amônia (Adaptado de Edgar e Himmelblau,1989).

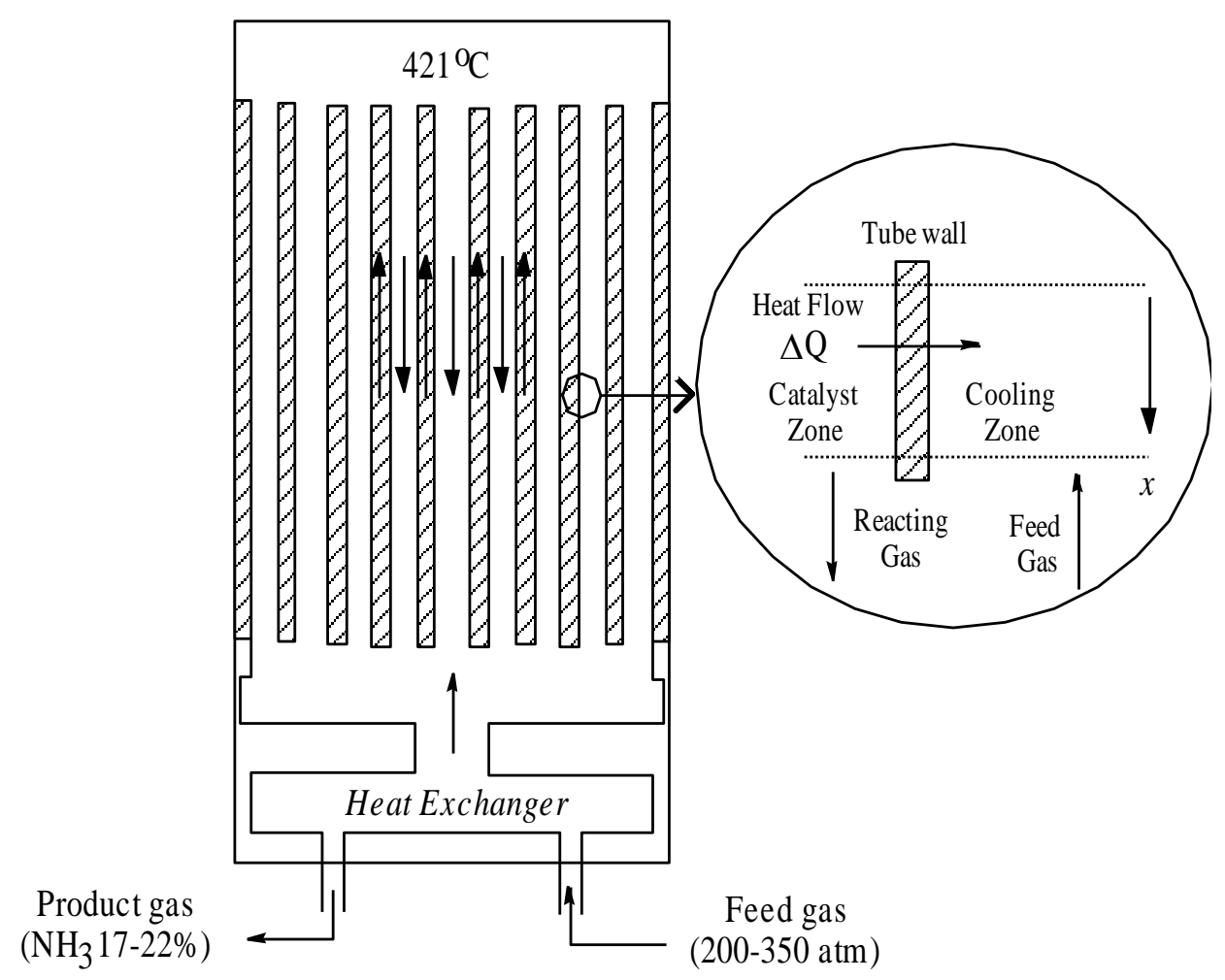

O projeto do reator apresentado neste trabalho enfatiza a maximização do lucro, baseado na diferença entre o valor do gás de produto e o valor do gás de alimentação menos a amortização dos custos de capital do reator (MURASE et al., 1970; UPRETI e DEB, 1997; EDGAR e HIMMELBLAU, 1989). Matematicamente, este objetivo é formulado como segue: 


$$
\begin{aligned}
& f\left(x_{L}, N_{N_{2}}, T_{f}, T_{g}\right)=1,33563 \times 10^{7}-1,70843 \times 10^{4} N_{N_{2}}+704,09\left(T_{g}-T_{0}\right)+ \\
& -699,27\left(T_{f}-T_{0}\right)-\left(3,45663 \times 10^{7}+1,98365 \times 10^{9} x_{L}\right)^{0,5}
\end{aligned}
$$

Neste caso, o vetor de funções objetivo é função de quatro variáveis: o comprimento do reator $\left(x_{L}\right)$, o número de moles de nitrogênio $\left(N_{N 2}\right)$, a temperatura do gás de reação $\left(T_{g}\right)$, e da temperatura do gás de alimentação $\left(T_{f}\right)$, para uma dada temperatura de referência $\left(T_{0}\right)$. Matematicamente, o modelo que descreve o processo acima é dado pelos balanços de massa e energia (YUSUP et al., 2006; KSASY et al., 2006):

$$
\begin{aligned}
& \frac{d T_{f}}{d x}=\frac{U S_{1}}{W C_{p f}}\left(T_{g}-T_{f}\right) \\
& \frac{d T_{g}}{d x}=-\frac{U S_{1}}{W C_{p g}}\left(T_{g}-T_{f}\right)+\frac{(-\Delta H) S_{2}}{W C_{p g}}\left(-\frac{d N_{N_{2}}}{d x}\right) \\
& \frac{d N_{N_{2}}}{d x}=-f_{a}\left(k_{1} \frac{p_{N_{2}}-p_{H_{2}}^{1,5}}{p_{N H_{3}}}-k_{2} \frac{p_{N_{3}}}{p_{H_{2}}^{1,5}}\right) \\
& k_{1}=1,79954 \times 10^{4} \exp \left(\frac{-20800}{R T_{g}}\right) \\
& k_{2}=2,5714 \times 10^{16} \exp \left(\frac{-47400}{R T_{g}}\right) \\
& p_{N_{2}}=\frac{286 N_{N_{2}}}{2,598 N_{N_{20}}+2 N_{N_{2}}} \\
& p_{H_{2}}=3 p_{N_{2}} \\
& p_{N_{3}}=\frac{286\left(2,23 N_{N_{20}}-2 N_{N_{2}}\right)}{2,598 N_{N_{20}}+2 N_{N_{2}}} \\
& 0 \leq N_{N_{2}} \leq 3220,400 \leq T_{f} \leq 800, \quad 0 \leq x_{L} \leq 10
\end{aligned}
$$

em que $U$ é o coeficiente global de transferência de calor (500 kcal/m² $\mathrm{h} \mathrm{K}$ ); $S_{1}$ é a área de superfície dos tubos de resfriamento por unidade de comprimento do reator $(10 \mathrm{~m})$; $W$ é o fluxo mássico $(26400 \mathrm{~kg} / \mathrm{h}) ; C_{p f}$ é a capacidade térmica do gás de alimentação $(0,707$ $\mathrm{kcal} / \mathrm{kg} \mathrm{K}) ; \Delta H$ é o calor de reação (-26000 kcal/kg moles de $\left.\mathrm{N}_{2}\right)$; $S_{2}$ é a área da seção transversal da zona do catalisador $\left(0,78 \mathrm{~m}^{2}\right) ;-d N_{N 2} / d x$ é a taxa de reação $(\mathrm{kg}$ moles de $\left.\mathrm{N}_{2} / \mathrm{hm}^{3}\right) ; C_{p g}$ é a capacidade térmica do gás de reação $(0,719 \mathrm{kcal} / \mathrm{kg} \mathrm{K}) ; f_{a}$ é a atividade do catalisador (1); $p_{N 2}, p_{H 2}$ e $p_{N H 3}$ são as pressões parciais de $N_{2}, H_{2}$ e $N_{3}$, respectivamente; $k_{1}$ e $k_{2}$ são as constantes de taxa; $R$ é a constante de gás ideal $(1,987$ $\mathrm{kcal} /(\mathrm{kg} \mathrm{mol} \mathrm{K})$; e $T_{0}$ é a temperatura de referência $(\mathrm{K})$. As condições de contorno 
associados ao sistema diferencial são: $T_{f}(x=0)=T_{0}, T_{g}(x=0)=T_{f}, N_{N 2}(x=0)=701,2 \mathrm{kmol} / \mathrm{m}^{2} \mathrm{~h}$ (Edgar e Himmelblau, 1989; Yusup et al., 2006; Ksasy et al., 2006).

\section{WHALE OPTIMIZATION ALGORITHM}

Como mencionado anteriormente, o WOA é um algoritmo evolutivo baseado no comportamento social de baleias jubarte, proposto para resolver problemas de otimização. Como mencionado por Mirjalili e Lewis (2016) e por Aljarah et al. (2016), uma baleia jubarte movimenta-se em uma trajetória em espiral em torno das suas presas, bem como cria bolhas ao longo desta trajetória, como observado na natureza. Como outras abordagens evolutivas, o WOA trabalha com uma população de potenciais candidatos que são atualizados dinamicamente ao longo do processo evolutivo. Inicialmente, uma população (com NP candidatos em potencial - baleias) é gerada aleatoriamente considerando o espaço de projeto definido pelo usuário. A seguir, os operadores (presas circundantes, neutralização de bolhas e busca por presas) considerados na WOA são aplicados para gerar uma nova população. Esse procedimento continua até que o número máximo de gerações seja encontrado. Estes operadores são descritos como segue.

\subsection{Operador de Presas Circundantes}

Neste operador, para atualizar a posição de uma baleia jubarte no espaço de projeto, a melhor solução corrente é a "presa alvo". Uma vez que o melhor candidato é definido, os outros candidatos de busca tentam atualizar suas posições em relação a essa presa (melhor solução da geração corrente). Este procedimento é definido de acordo com as seguintes equações:

$$
\begin{aligned}
& D=\left|C X^{*}(I)-X(I)\right| \\
& X(I+1)=X^{*}(I)-A D
\end{aligned}
$$

onde $D$ é a distância entre a melhor solução (corrente) $X^{*}$ e uma solução $X$ na $l$-ésima geração, $C$ é um parâmetro definido como $2 r$ ( $r$ é um vetor de números aleatórios definidos no intervalo $[0,1])$, $A$ é igual a $2 a r-a$ (a decresce linearmente de 2 à 0 ao longo das gerações).

\subsection{Operador de Neutralização das Bolhas}

Conforme mencionado por Mirjalili e Lewis (2016), este operador apresenta dois mecanismos básicos: o encolhimento encurvado e o posicionamento em espiral, 
responsáveis pela atualização dos candidatos da população. No primeiro mecanismo, $A$ é um valor aleatório no intervalo [-a, a], que é reduzido ao longo das gerações. Definindo valores aleatórios para $A$ em [-1, 1], a nova posição de um candidato de busca pode ser definida em qualquer lugar entre a posição original e a posição do melhor candidato da geração corrente (ALJARAH et al., 2016). No segundo mecanismo, propõe-se uma relação em espiral para imitar o movimento em forma de hélice de baleias jubarte para gerar uma nova posição. Esta relação é definida como:

$$
X(I+1)=D^{\prime} \exp (b \gamma) \cos (2 \pi \gamma)+X^{*}(I)
$$

em que $D^{\prime}=\left|X^{*}(I)-X(I)\right|$ representa a distância da l-ésima baleia da presa na l-ésima geração, $b$ é uma constante que define a forma da espiral logarítmica. $\gamma$ é um parâmetro definido em função de um outro parâmetro, a saber, a2, $\gamma=\left(a_{2}-1\right)$ rand+1 e que decresce linearmente de -2 à -1 ao longo das gerações, onde rand é um gerador de números aleatórios pertencentes ao intervalo [-1 1 ].

Para descrever a forma como as baleias jubarte nadam em torno da presa (dentro de um círculo que encolhe) e ao longo de um caminho em forma de espiral, assume-se a seguinte probabilidade (50\% de chance de encolher o mecanismo circundante e $50 \%$ de chance para a atualizar a posição das baleias):

$$
X(I+1)= \begin{cases}X^{*}(I)-A D & \text { se } p<0,5 \\ D^{\prime} \exp (b \gamma) \cos (2 \pi \gamma)+X^{*}(I) & \text { se } p \geq 0,5\end{cases}
$$

onde $p$ é um número aleatório pertencente ao domínio $[0,1]$.

De acordo com Mirjalili e Lewis (2016), na equação acima, o primeiro termo representa o mecanismo circundante e o segundo simula o procedimento de "neutralização de bolhas". A utilização do parâmetro p garante a alternância entre estas duas fases com uma mesma probabilidade. As prováveis posições de um candidato considerando esta estratégia são ilustradas na Figura 2. 
Figura 2. Mecanismos de encolhimento e neutralização das bolhas consideradas para atualizar potenciais candidatos usando o WOA (Adaptado de Mirjalili e Lewis, 2016).
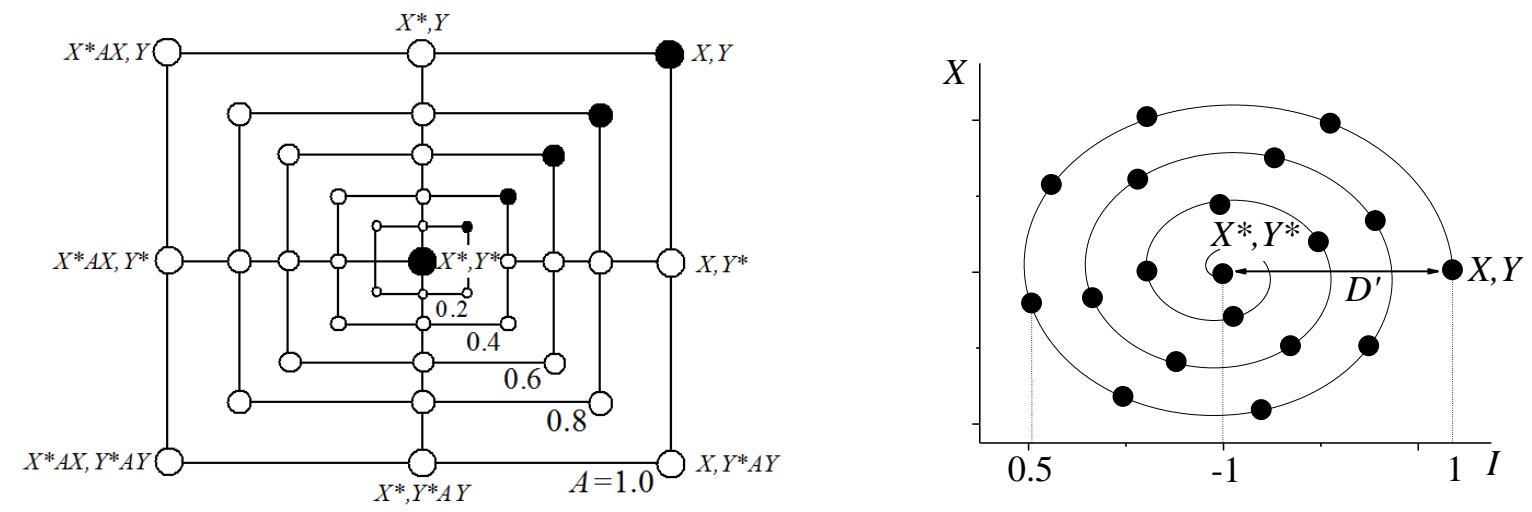

\subsection{Operador de Busca pela Presa}

Nesta estratégia de otimização heurística, os mecanismos de exploração se devem à atualização dos parâmetros a e $C$ em cada geração. Em cada geração, a população de candidatos atualiza suas posições com base em um candidato selecionado aleatoriamente ou o melhor agente de busca obtido até agora. Conforme mencionado por Aljarah et al. (2016), a fim de garantir os mecanismos de exploração e convergência, a melhor solução é o ponto pivô para atualizar a posição de outros candidatos quando $|A|>$ 1. Por outro lado, a melhor solução obtida até agora desempenha o papel do ponto de articulação.

$\mathrm{Na}$ literatura, algumas aplicações considerando esta nova estratégia podem ser encontradas. Mirjalili e Lewis (2016) aplicaram o WOA para a otimização de funções matemáticas com diferentes níveis de complexidade e no projeto de sistemas de engenharia clássicos. Rohani et al. (2016) aplicaram o WOA para o planejamento do fluxo de trabalho em canteiros de obras. Prakash e Lakshminarayana (2016) empregaram o WOA para encontrar o dimensionamento e o posicionamento ótimo de capacitores em um sistema de distribuição radial. Hu et al. (2016) propuseram uma nova metodologia para atualizar um dos parâmetros (peso de inércia) no WOA. O desempenho deste novo algoritmo foi avaliado durante a otimização de funções matemáticas. Neste mesmo contexto, Trivedi et al. (2016) também propuseram uma nova técnica para aumentar a taxa de convergência em WOA. Essa abordagem foi testada em dez funções matemáticas clássicas. Aljarah et al. (2016) aplicaram o WOA para a otimização dos pesos de conexão em redes neurais. 


\section{METODOLOGIA}

A metodologia proposta neste trabalho consiste na utilização do WOA para o projeto de um reator de amônia. Além disso, apresentar uma forma alternativa para a atualização dinâmica de dois parâmetros do WOA, a saber, a e a2, respectivamente. Conforme sugerido por Mirjalili e Lewis (2016), ambos os parâmetros são atualizados de forma linear, conforme definido a seguir:

$$
\begin{aligned}
& a=2-k\left(\frac{2}{N_{g e n}}\right) \\
& a_{2}=-1+k\left(\frac{-1}{N_{g e n}}\right)
\end{aligned}
$$

em que $k$ é a geração corrente e $N_{\text {gen }}$ é o número máximo de gerações permitida.

A principal desvantagem desta abordagem é o fato da mesma independer de quaisquer informações sobre o andamento do processo evolutivo, isto é; a mesma é apenas uma relação empírica proposta por Mirjalili e Lewis (2016) a partir de ampla experimentação numérica.

Como alternativa para a atualização destes parâmetros, no presente estudo é considerado o conceito de taxa de convergência como mecanismo para o qual ambos os parâmetros serão atualizados. Neste contexto, considere o conceito de taxa de convergência $(T C)$ conforme a seguinte relação:

$$
T C=\frac{f_{\text {average }}}{f_{\text {worst }}}
$$

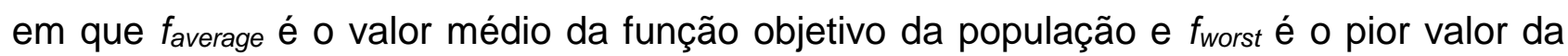
função objetivo da população, respectivamente.

A TC pode ser interpretada como uma forma de mensurar a homogeneidade da população em um processo evolutivo. Inicialmente tem-se TC próximo de zero, i.e., o "pior" valor da função objetivo é bem diferente do valor médio da função objetivo, o que faz com que a taxa seja pequena. Já no final do processo evolutivo, como a população tende a ficar homogênea, TC tende a 1.

Definida a taxa de convergência, pode-se propor a seguinte relação para a atualização dos parâmetros a e a2:

$$
a=2-2(1-T C)
$$




$$
a_{2}=-1-T C
$$

onde a e a2 variam nos intervalos [0 2] e [-2 -1], respectivamente, conforme sugerido por Mirjalili e Lewis (2016). Todavia, os mesmos são atualizados a partir da evolução do valor da função objetivo a partir do conceito de taxa de convergência e não como uma relação puramente empírica.

\section{RESULTADOS E DISCUSSOEES}

Para avaliar o desempenho da metodologia proposta e denominada de Adaptive Whale Optimization Algorithm (AWOA) para o projeto de um reator de amônia considerase o algoritmo original (WOA) com os parâmetros a e a2 sendo atualizados conforme sugerido por Mirjalili e Lewis (2016). Além disso, dois estudos de caso são considerados considerando diferentes valores para $T_{0}(694 \mathrm{~K}$ e $800 \mathrm{~K})$. Os outros parâmetros considerados nas simulações são descritos a seguir:

- Objetivo: maximização do lucro;

- Espaço de Projeto (MURASE et al., 1970; UPRETI e DEB, 1997; EDGAR e HIMMELBLAU, 1989): 0,01 $\mathrm{m} \leq \mathrm{x} \leq 10 \mathrm{~m} ; 400 \mathrm{~K} \leq T_{f} \leq 800 \mathrm{~K} ; 500 \mathrm{~K} \leq T_{g} \leq 700 \mathrm{~K}$ e 0 $\mathrm{kmol} / \mathrm{m}^{2} \mathrm{~h} \leq \mathrm{N}_{\mathrm{N} 2} \leq 700 \mathrm{kmol} / \mathrm{m}^{2} \mathrm{~h}$;

- Parâmetros considerados nos algoritmos: 50 indivíduos (baleias) na população (WOA e AWOA), 500 gerações (WOA e AWOA), e os parâmetros a e a2 sendo atualizados conforme as Equações (15) e (16) no WOA e os parâmetros a e a2 sendo atualizados conforme as Equações (18) e (19) no AWOA;

- É importante ressaltar que, para os parâmetros considerados, ambos os algoritmos requerem 50+50×500 avaliações da função objetivo;

- Cada algoritmo foi executado 10 vezes para a obtenção dos valores que são apresentados na tabela que se segue.

\subsection{Considerando $T_{0}=694 \mathrm{~K}$}

A Tabela 1 apresenta os resultados obtidos pelos algoritmos WOA e AWOA considerando $T_{0}$ igual a $694 \mathrm{~K}$. Nesta tabela é possível observar que o valor da temperatura do gás de alimentação $\left(T_{f}\right)$ atingiu o seu valor mínimo (em $X=X L$ ) para a obtenção da maximização do lucro. Já para os valores referentes ao número de moles de nitrogênio $\left(N_{N 2}\right)$ e da temperatura do gás de reação $\left(T_{g}\right)$, foram obtidos valores médios, conforme o espaço de projeto considerado. Em termos físicos, os resultados 
apresentados estão em concordância com aqueles reportados por Babu e Angira (2005) e Yusup et al. (2006). Como destacado por Babu e Angira (2005), os resultados reportados por Edgar et al. (2001) e Upreti e Deb (1997) são distintos porque o modelo considerado por estes autores apresenta erros relacionados à modelagem do processo físico.

Tabela 1. Resultados obtidos pelos algoritmos WOA e AWOA para a $T_{0}=694 \mathrm{~K}$.

\begin{tabular}{cccccc}
\hline Referência & $\boldsymbol{x}_{L}(\mathbf{m})$ & $\boldsymbol{T}_{\boldsymbol{f}}(\mathrm{K})$ & $\boldsymbol{T}_{\boldsymbol{g}}(\mathrm{K})$ & $\boldsymbol{N}_{\boldsymbol{N} 2}\left(\mathrm{Kmol} / \mathbf{m}^{2} \mathbf{h}\right)$ & $\boldsymbol{f} \mathbf{x} \mathbf{1 0 ^ { - 6 }}(\mathbf{\$})$ \\
\hline Edgar et al. (2001) & 2,58 & - & - & - & 1,29 \\
\hline Upreti e Deb (1997) & 5,33 & - & - & - & 4,23 \\
\hline Babu e Angira (2005) & 6,58 & - & - & - & 5,00 \\
\hline Yusup et al. (2006) & 6,695 & 400 & 629,65 & 490,84 & 5,015 \\
\hline WOA & $6,696^{1}$ & 400 & 629,71 & 490,79 & 5,056 \\
& $6,697^{2}$ & 400 & 629,73 & 490,79 & 5,026 \\
& $6,694^{3}$ & 400 & 629,74 & 490,79 & 5,001 \\
\hline AWOA & $6,695^{1}$ & 400 & 629,71 & 490,79 & 5,056 \\
& $6,696^{2}$ & 400 & 629,71 & 490,81 & 5,020 \\
& $6,696^{3}$ & 400 & 629,71 & 490,88 & 5,010 \\
\hline
\end{tabular}

Fonte: Elaborado pelos autores.

Legenda: ${ }^{1}$ Best, ${ }^{2}$ Average $\mathrm{e}^{3}$ Worst.

A Figura 3 apresenta os perfis ótimos para o fluxo mássico e para as temperaturas no reator considerando $T_{0}$ igual a $694 \mathrm{~K}$. Nesta figura percebe-se que o fluxo molar de nitrogênio, como esperado, diminui com relação ao comprimento do reator até atingir um valor limite (limitante de reação). O perfil de temperatura do gás de reação inicialmente aumente até um valor ótimo e logo em seguida diminui. Já o perfil de temperatura do gás de alimentação sempre diminui ao longo do comprimento do reator.

\subsection{Considerando $T_{0}=800 \mathrm{~K}$}

A Tabela 2 apresenta os resultados obtidos por ambos os algoritmos considerando $T_{0}$ igual a $800 \mathrm{~K}$. Conforme observado nesta tabela, os resultados obtidos estão em concordância com aqueles reportados por Borges et al. (2012) considerando o Algoritmo de Busca Gravitacional. 


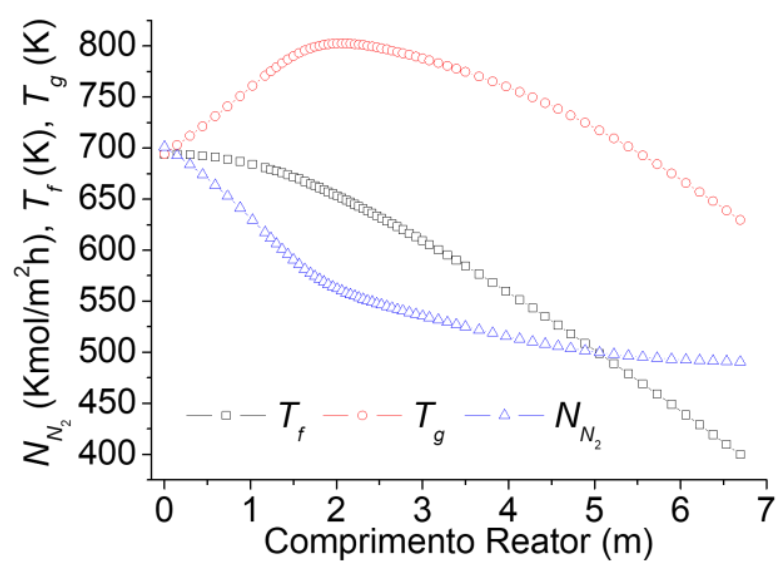

Figura 3. Perfis para o fluxo mássico e de temperaturas no reator para a $T_{0}=694 \mathrm{~K}$.

Tabela 2. Resultados obtidos pelos algoritmos WOA e AWOA para a $T_{0}=800 \mathrm{~K}$.

\begin{tabular}{cccccc}
\hline Referência & $\boldsymbol{x}_{\boldsymbol{L}}(\mathbf{m})$ & $\boldsymbol{T}_{\boldsymbol{f}}(\mathrm{K})$ & $\boldsymbol{T}_{\boldsymbol{g}}(\mathrm{K})$ & $\boldsymbol{N}_{\boldsymbol{N} \mathbf{2}}\left(\mathrm{Kmol} / \mathbf{m}^{2} \mathbf{h}\right)$ & $\boldsymbol{f \times \mathbf { 1 0 } ^ { - 6 } ( \mathbf { \$ } )}$ \\
\hline Borges et al. (2012) & 9,515 & 400 & 631,28 & 490,96 & 4,991 \\
\hline \multirow{2}{*}{ WOA } & $9,515^{1}$ & 400 & 631,29 & 490,96 & 4,991 \\
& $9,515^{2}$ & 400 & 631,29 & 490,96 & 4,991 \\
& $9,516^{3}$ & 400 & 631,29 & 490,97 & 4,993 \\
\hline \multirow{2}{*}{ AWOA } & $9,515^{1}$ & 400 & 631,29 & 490,97 & 4,991 \\
& $9,516^{2}$ & 400 & 631,29 & 490,97 & 4,992 \\
& $9,516^{3}$ & 400 & 631,29 & 490,97 & 4,992 \\
\hline
\end{tabular}

Fonte: Elaborado pelos autores.

Legenda: ${ }^{1}$ Best, ${ }^{2}$ Average $\mathrm{e}^{3}$ Worst.

Nesta tabela observa-se que, em relação ao estudo de caso anterior $\left(T_{0}=694 \mathrm{~K}\right)$, o valor da temperatura do gás de alimentação também atingiu o seu valor mínimo, o número de moles de nitrogênio foi ligeiramente superior e o valor da temperatura do gás de reação foi praticamente igual ao encontrado para $T_{0}=694 \mathrm{~K}$.

A Figura 4 apresenta os perfis ótimos para o fluxo mássico e para as temperaturas no reator considerando $T_{0}$ igual a $800 \mathrm{~K}$. Assim como na figura anterior, percebe-se que o fluxo molar de nitrogênio diminui com ao aumento do tamanho do comprimento do reator até atingir um valor limite (limitante de reação). Já o perfil de temperatura do gás de reação aumenta rapidamente até um valor ótimo e logo em seguida diminui. Já o perfil de temperatura do gás de alimentação sempre diminui ao longo do comprimento do reator. É importante ressaltar que, diferente do estudo de caso anterior, os valores das temperaturas do gás de alimentação e de reação apresentam valores superiores aos 
considerados para $T_{0}=694 \mathrm{~K}$. Neste caso, o processo inicializa-se a uma temperatura superior a considerada no estudo de caso anterior.

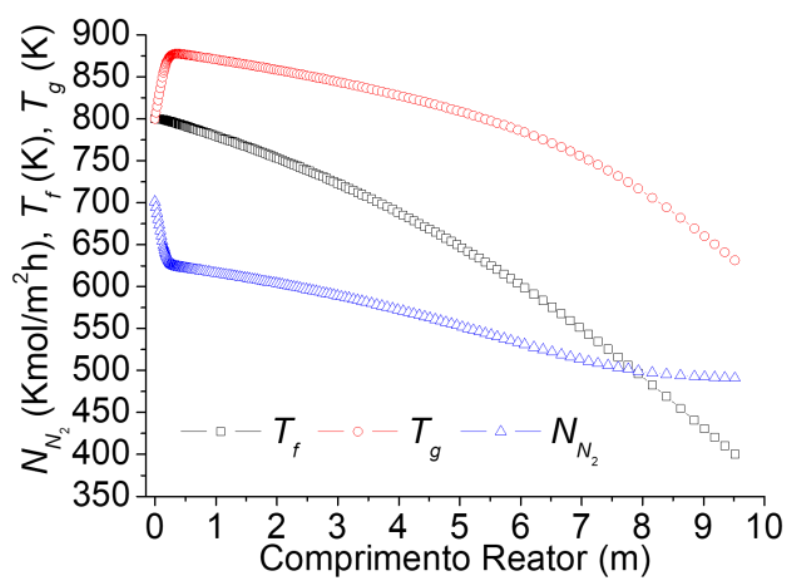

Figura 4. Perfis para o fluxo mássico e de temperaturas no reator para a $T_{0}=800 \mathrm{~K}$.

\section{CONSIDERAÇOES FINAIS}

O presente trabalho teve como objetivo o projeto de um reator de amônia através da determinação de condições de operação para fins da maximização deste processo. Para essa finalidade foi considerado o Whale Optimization Algorithm. Neste sentido, uma nova proposta baseada no conceito de taxa de convergência para a atualização dinâmica de dois parâmetros deste algoritmo foi proposta. Os resultados obtidos demonstraram que ambos os algoritmos foram capazes de encontrar a solução apresentada na literatura especializada, i.e., a proposta para a atualização dinâmica dos parâmetros foi equivalente, em termos do valor da função objetivo, à proposta por Mirjalili e Lewis (2016). Todavia, cabe ressaltar que, diferentemente da metodologia proposta por Mirjalili e Lewis (2016), a desenvolvida neste trabalho é baseada na evolução do valor da função objetivo, sendo neste caso, função do problema de otimização a ser resolvido.

Como proposta para trabalho futuro pode-se citar a extensão deste algoritmo para o contexto multi-objetivo, bem como a sua aplicação para a resolução de problemas considerando robustez e confiabilidade.

\section{REFERÉNCIAS}

ALJARAH, I, FARIS, H., MIRJALILI, S. Optimizing Connection Weights in Neural Networks using the Whale Optimization Algorithm. Soft Computing, DOI 10.1007/s00500-016-24421, 1-15, 2016. 
ANNABLE, D. Application of the Temkin Kinetic Equation to Ammonia Synthesis in the Large-Scale Reactors, Chemical Engineering Science, 1(4), p. 145-150, 1952.

BABU, B. V., ANGIRA, R. Optimal Design of an Auto-thermal Ammonia Synthesis Reactor, Computers and Chemical Engineering Journal, v. 29, p. 1041-1045, 2005.

BABU, B.V., ANGIRA, R., NILEKAR, A, Optimal Design of an Auto-Thermal Ammonia Synthesis Reactor Using Differential Evolution, Proceedings of The Eighth World MultiConference on Systemics, Cybernetics and Informatics (SCI-2004), Orlando, Florida, USA, July, p. 18-21, 2004.

DYSON, D. C. Optimal Design of Reactors for single Exothermic Reversible Reactions, Ph.D. thesis, London University, 1965.

EDGAR, T. F., HIMMELBLAU, D. M. Optimization of Chemical Processes. New York, McGraw-Hill, 1989.

HU, H., BAI, Y., XU, T. A Whale Optimization Algorithm with Inertia Weight. WSEAS Transactions on Computers, (15), p. 319-326. E-ISSN: p. 2224-2872, 2016.

KSASY, M. S. M., AREED, F., SARAYA, S., KHALIK, M. A. Optimal Reactor Length of an Auto-Thermal Ammonia Synthesis Reactor. International Journal of Electrical \& Computer Sciences, 10(3), p. 1-7, 2006.

MIRJALILI, S., LEWIS, A. The Whale Optimization Algorithm. Advances in Engineering Software, v. 95, p. 51-67, 2016.

MURASE, A., ROBERTS, H. L., CONVERSE, A. O. Optimal Thermal Design of an Autothermal Ammonia Synthesis Reactor, Industrial \& Engineering Chemistry Research, v. 9, p. 503-513, 1970.

PRAKASH, D.B., LAKSHMINARAYANA, C. Optimal Siting of Capacitors in Radial Distribution Network. Alexandria Engineering Journal, doi.org/10.1016/j.aej.2016.10.002, 2016.

ROHANI, M., SHAFABAKHSH, G., HADDAD, A., ASNAASHARI, E. The Workflow Planning of Construction Sites using Whale Optimization Algorithm, The Turkish Online Journal of Design, Art and Communication, DOI NO: 10.7456/1060NVSE/107, p. 29382951, 2016.

TRIVEDI, I.N., PRADEEP, J., NAROTTAM, J., ARVIND, K., DILIP, L. A Novel Adaptive Whale Optimization Algorithm for Global Optimization. Indian Journal of Science and Technology, 9(38), 1-6, DOI: 10.17485/ijst/2016/v9i38/101939, 2016.

UPRETI, S. R., DEB, K. Optimal Design of an Ammonia Synthesis Reactor using Genetic Algorithms, Computers \& Chemical Engineering, v. 21, p. 87-92, 1997.

YUSUP, S., ZARIRI, H., YUSOFF, N., YEW, Y. C. Modeling and Optimization of Ammonia Reactor using Shooting Methods, Proceedings of the 5th WSEAS Int. Conf. on Data Networks, Communications \& Computers, Bucharest, Romania, October p. 16-17, 2006. 